\title{
Aprendizaje Basado en Problemas: Seminario Integrado en el Grado de Enfermería
}

\author{
José Granero-Molina*, Cayetano Fernández-Sola, Adelaida Mª Castro-Sánchez \\ y Gabriel Aguilera-Manrique \\ Universidad de Almería, Departamento de Enfermería y Fisioterapia, Facultad de Cs. de la Salud, \\ Carretera Sacramento, S/No, (04120) La Cañada de San Urbano, Almería-España. \\ (email: jgranero@ual.es) \\ * Autor a quien debe ser dirigida la correspondencia
}

Recibido Mar. 29, 2011; Aceptado Abr. 28, 2011; Versión final recibida May.02, 2011

\begin{abstract}
Resumen
Este trabajo presenta y analiza las experiencias del alumnado en el desarrollo de un seminario integrado, mediante la metodología denominada Aprendizaje Basado en Problemas (ABP). La experiencia se realiza en asignaturas de enfermería de la Facultad de Ciencias de la Salud de la Universidad de Almería en España. El estudio se basa en la Teoría Fundamentada en Datos que emplea el método inductivo para descubrir teorías, conceptos e hipótesis, partiendo directamente de los datos. La integración teoría-práctica, el aprendizaje colaborativo y la relevancia de los tutores, son los aspectos más valorados, aunque también surgen opiniones discrepantes sobre su utilidad en la adquisición de ciertas competencias. La experiencia es altamente positiva pero se requiere una planificación más profunda, previa a la puesta en práctica de nuevas metodologías de enseñanza y evaluación como el ABP.
\end{abstract}

Palabras clave: aprendizaje basado en problemas, conocimiento crítico, métodos de enseñanza, enfermería

\section{Problem Based Learning: Integrated Seminar in Nursing Degree}

\begin{abstract}
This study presents and analyzes the experiences of students in the development of an integrated seminar by a methodology called Problem Based Learning (PBL). This experience is carried out by nursing students of the Faculty of Health Sciences at the University of Almeria (Spain). The study was based on Grounded Theory which uses the inductive method for discovering theories, concepts and hypotheses starting directly from the data. The integration of theory and practice, collaborative learning and the relevance of the tutors are the most valued, but divergent views also arise about its utility in the acquisition of certain competences. The experience is very positive but a deeper planning is required before the implementation of new teaching methodologies and assessment such as PBL.
\end{abstract}

Keywords: problem-based learning, critical thinking, teaching methods, nursing studies 


\section{INTRODUCCIÓN}

La Unión Europea trata de armonizar las políticas conducentes al bienestar de los ciudadanos de sus estados miembros, la creación del Espacio Europeo de Educación Superior (EEES) supone un esfuerzo por unificar los sistemas educativos (Fernández y Granero, 2008). En esa línea, se produce la renovación de las metodologías docentes, potenciando aquellas que den respuesta al carácter teórico-práctico del conocimiento y, todo ello, inscrito en una apuesta por la mejora de la calidad (Cancela et al., 2010). Este artículo describe la puesta en práctica del Aprendizaje Basado en problemas (ABP) en CCSS, mediante el desarrollo de un "seminario integrado" en el que participan 5 asignaturas de la Titulación de Enfermería. Esta metodología educativa, centrada en el estudiante, es clave en el logro de competencias aplicables a entornos técnicos y prácticos, donde confluyen el conocimiento empírico y el interpretativo (Giuliano, 2003). Su descripción, junto al análisis de las experiencias del alumnado participante, contribuyen a fomentar la discusión acerca de su aplicación y validez en áreas de conocimiento donde el factor humano, inherente al proceso salud-enfermedad, sigue siendo clave.

Amparada en la racionalidad científico-técnica, la acción transformadora del hombre sobre el mundo se erige en base del conocimiento y eje del progreso en la modernidad (Jonas, 1997). Frente a las ciencias naturales, donde prima la explicación de los fenómenos en un mundo de causas y consecuencias probabilísticamente predecibles, las CCSS presentan la conflictividad de derivar de la generalidad el caso particular del individuo que enferma. Las cuestiones particulares no se domeñan a la generalización, las experiencias escapan de la metodología empírico-analítica y la realidad excede a la explicación teórica. Frente a la obcecación metodológica de la ciencia, la praxis reclama un espacio en las CCSS donde la opinión, el juicio clínico y la particularidad de cada ser humano complementen los datos objetivos. Esta singularidad de las CCSS se refleja tanto en los métodos de investigación como en los de enseñanza-aprendizaje. Frente a modelos de reflexión técnicos, que aplican rigurosamente la teoría científica, el modelo práctico define medios y fines según las situaciones problemáticas de la vida real (Schön, 1998). La "deficiente conexión teoría-práctica" en estas áreas de conocimiento, donde se engloban estudios como medicina, enfermería, odontología, o Fisioterapia, obstaculiza el cambio en las organizaciones educativas; pues la escasa relación entre las condiciones del aula y las de la vida real convierten en "especialmente dificultosa" la adquisición de competencias. En este sentido, si bien la currícula del Grado en Enfermería incorpora conocimientos teóricos, teórico-prácticos y prácticas clínicas; sigue echándose en falta una apuesta más decidida por métodos que interrelacionen e imbriquen estos tipos de conocimiento.

La clase expositiva tradicional ya no ofrece todas las respuestas para el desarrollo de habilidades técnicas, de juicios clínicos y de la capacidad de resolución de problemas; una cuestión que conduce a incorporar nuevas metodologías, como el ABP, en las Facultades de CCSS (Hodges, 2011). La psicología cognitiva proporciona una base teórica al ABP, sustentado en la teoría constructivista sobre el aprendizaje humano que une comprensión, conocimiento previo y contexto al enfrentar las situaciones reales (Spencer, 2003). El ABP se define como "un método de aprendizaje basado en el principio de usar problemas como punto de partida para la adquisición e integración de nuevos conocimientos" (Barrows, 1986). El problema del paciente requiere del estudiante razonamiento clínico y pensamiento crítico, por eso el ABP incluye trabajo en grupos pequeños, el profesor como guía del proceso, los problemas como vehículo para el desarrollo de habilidades, los estudiantes como determinantes de las necesidades de aprendizaje, el estudio individual, el análisis grupal y la síntesis final (Morales y Landa, 2004).

Preocupados por la reducción de la brecha teoría-práctica en los estudiantes de la Facultad de Ciencias de la Salud de la Universidad de Almería (UAL), y en el marco de la experiencia piloto del EEES, el equipo docente de tercer curso diseñó un "seminario integrado", donde participan las asignaturas de Enfermería Comunitaria III, Enfermería Geriátrica, Enfermería Psiquiátrica, Enfermería-Médico-Quirúrgica y Metodología del cuidado en el estudio y resolución de un caso común: "Mujer de 87 años, que ingresa en una unidad de traumatología. Se la interviene de una fractura de cadera izquierda, a los dos días presenta signos de osteomielitis, pérdidas de orina previo a la intervención, que aumentaban con la presión intraabdominal. Tras la retirada de la 
sonda vesical presenta episodios de incontinencia urinaria. En la actualidad continua con deterioro de la movilidad física. A las 24 horas de la intervención, presenta desorientación, delirios y alucinaciones, siendo dada de alta a su domicilio". El proceso implicó mantener dos reuniones previas para acordar "el caso" (problema), la metodología de trabajo y el reparto en grupos pequeños (Morales y Landa, 2004). Los 140 alumnos del curso se dividen en 14 grupos, realizando cada uno de ellos dos sesiones de 2 horas, guiado por un "profesor". Primera sesión: presentación del caso, análisis grupal del escenario del problema, lluvia de ideas para sugerir hipótesis sobre causas y soluciones.

Tras definir el problema, se confeccionan subgrupos (2-3 alumnos) para la búsqueda de información, análisis e interpretación de la misma. Tutoría de expertos: la semana siguiente los profesores realizan tutorías para que los alumnos consulten las dudas que presenta el caso con cada asignatura. Segunda sesión: el profesor plantea cada cuestión (problema) al hilo del caso y elije al azar a un alumno para que presente su respuesta individual, aunque el trabajo haya sido grupal; posteriormente, interviene el resto ampliando o matizando esa respuesta. La sesión finaliza con una autoevaluación en la que cada alumno evalúa su aportación individual, el trabajo del grupo, el trabajo del profesor y la metodología ABP. El objetivo principal del trabajo presentado en este artículo es conocer el significado que los alumnos participantes en un Seminario integrado atribuyen al ABP como metodología de enseñanza aprendizaje.

\section{METODOLOGÍA}

Se usa la Teoría Fundamentada en datos (TFD) (Glaser y Strauss, 1967), que sienta sus bases en el Interaccionismo Simbólico (Burbank y Martins, 2010). La TFD emplea el método inductivo para descubrir teorías, conceptos e hipótesis partiendo directamente de los datos. Se trata de un proceso dinámico basado la comparación constante, empleada desde el inicio del análisis hasta la elaboración de mapas conceptuales; y el muestreo teórico, al que se recurre tras las primeras 15 entrevistas para desarrollar las categorías "crítica a la metodología" y "relación teoría-práctica", y que implicó aumentar en tres el número de entrevistados. El punto de partida empírico de este enfoque está en los significados subjetivos que los individuos atribuyen a sus actividades y sus ambientes (Flick, 2009), luego la recogida de la información se realizó mediante una entrevista a 18 alumnos, a los que se les pide una crítica razonada sobre el seminario realizado. Una vez transcrita la entrevista, se guarda en formato RTF compatible con el programa ATLAS.ti 6.0®, generando una Unidad Hermenéutica analizada mediante reelaboración de las categorías iniciales y la adición de otras emergentes. Se identifican como Citas (Quotations) los fragmentos de respuesta más significativos. A cada cita se le asignan Códigos (Codes), mediante los procedimientos de Open coding (códigos construidos a partir de categorías predefinidas del guión de la entrevista) y Codes in vivo (códigos emergentes de palabras o frases de la entrevista). Cuando se han analizado 18 entrevistas, al no aparecer elementos nuevos en el análisis, los autores consideramos que se ha alcanzado la saturación teórica de los datos y que la información obtenida puede considerarse suficiente para los objetivos propuestos (Charmaz, 2006). Para eliminar el sesgo del investigador y aumentar la validez del estudio, dos colegas, de manera independiente, analizaron y discutieron las categorías encontradas, concluyendo con la emisión de un listado final. Seguidamente, se realiza una relectura de las transcripciones para comprobar si las categorías elegidas cubren todos los aspectos de las mismas (Kennedy y Lingard, 2006). En un segundo nivel interpretativo de los datos, se crean familias de códigos relevantes para la pregunta de investigación (Strauss y Corbin, 1990), comparando texto con códigos. En un tercer nivel interpretativo se establecen Network entre citas, códigos y/o familias de códigos, obteniendo representaciones gráficas de las interacciones entre los mismos. Todos los participantes en la entrevista fueron informados del propósito del estudio, del carácter voluntario de su participación, y del compromiso de mantener la confidencialidad y el anonimato. Se obtuvo el consentimiento informado de los participantes.

\section{RESULTADOS}

Se seleccionó por muestreo teórico a un total de 18 alumnos cuya edad oscila entre los 21 y 27 años, con una media de 22.6 años. De ellos 14 son mujeres y 4 varones. Los resultados se 
presentan agrupados en torno a 3 categorías emergentes fundamentales, apoyándose, a su vez, en la transcripción literal de respuestas codificadas como $\left(E_{1}, E_{2}, \ldots E_{n}\right)$.

Aprendizaje cooperativo: la valoración global del seminario es positiva, argumentando tanto la utilidad para el aprendizaje "La metodología del seminario la veo adecuada, hemos aprendido unos de otros" $\left(\mathrm{E}_{4}\right)$, como el mayor compromiso del estudiante: "Creo que con esta dinámica podemos aprender mejor y es un trabajo nuestro realizado con más compromiso." ( $\left.E_{9}\right)$. Mayoritariamente, los alumnos reclaman que se use más frecuentemente, señalando al debate como el aspecto que más les aporta pues; además de compartir conocimientos, "Supone recibir visiones distintas sobre las mismas situaciones, donde más he aprendido ha sido en el debate" $\left(E_{7}\right)$, les clarifica conceptos y dudas: "A la hora de debatirlo en clase, te ayuda en la aclaración de ideas." $\left(E_{11}\right)$. El papel del tutor se reconoce como esencial: "Sin la aportación del moderador que reconduce hacia lo esencial, el debate derivaría hacia cuestiones intrascendentes." $\left(\mathrm{E}_{3}\right)$

Relación teoría- práctica: reconocida como positiva por la mayoría de los alumnos (Ver Fig. 1), se evidencia más en el seminario que en otras metodologías docentes: "Se deberían hacer más a menudo para relacionar mejor los conceptos, la teoría no sirve de nada si no sabemos aplicarla" $\left(E_{1}\right)$. La aplicación del conocimiento va ligada a esta relación: "Nuestros conocimientos los hemos aplicado a un caso práctico, porque a lo largo del curso damos teoría que no se aplica a casos reales." $\left(E_{13}\right)$. El carácter integral del seminario ha sido señalado por varios alumnos $(n=3)$, destacando la transversalidad del conocimiento: "Integramos todas la materias y las utilizamos de forma transversal en un caso práctico." $\left(\mathrm{E}_{18}\right)$

Crítica a la metodología: para algunos alumnos $(n=4)$ esta metodología no aporta nada nuevo, "El debate aporta poco, la verdad. Pocas ideas nuevas, lo de siempre." $\left(E_{2}\right)$, no encontrándole ventajas con respecto a otras metodologías: "Bajo mi punto de vista, se aprende igual que cualquier otra clase magistral." $\left(E_{11}\right)$. En ocasiones, tener que realizar un trabajo resta valor al seminario y, en la valoración negativa del alumno subyace un rechazo al trabajo autónomo: "La dinámica de trabajo no es buena, espero que se valore, porque le hemos dedicado mucho tiempo entre todos." $\left(E_{12}\right)$. La crítica más repetida $(n=8)$ es la cercanía del seminario a los exámenes, reclamando la suspensión de la actividad académica y cualquier otra actividad adicional durante este el período: "No estoy de acuerdo en la fecha del seminario integrado, tendría que haberse realizado antes, no ahora que estamos cerca de los exámenes." $\left(E_{1}\right)$. También aflora la preocupación porque la evaluación del seminario no refleje el tiempo de dedicado al mismo (Ver Fig. 2): "Supone una carga muy importante de trabajo en muy poco espacio de tiempo, ... que esperemos sea valorada." ( $\left.E_{5}\right)$. La repetición del mismo caso que en el curso pasado es percibida como negativa y desmotivadora en repetidores de curso: "Reconozco una desmotivación por tener que repetir el mismo ejercicio que ya trabajé con el grupo del curso pasado" $\left(E_{15}\right)$. La autoevaluación más común es de "notable", expresado numéricamente y de forma razonada.

\section{DISCUSIÓN}

Es necesario un cambio curricular que de soluciones a la falta de conexión entre los conocimientos teóricos adquiridos en la universidad, y las competencias requeridas para la práctica (Zuberi, 2011). En esta línea, las CCSS deben ofrecer respuestas a situaciones complejas, más allá de la aplicación de conocimientos sistematizados, integrando el binomio teoría-práctica mediante metodologías como el ABP (Wood, 2008). Este enfoque, muy extendido para el aprendizaje en ambientes tecnológicos (Chunta y Katrancha, 2010), es valorado porque facilita el desempeño del rol y los conocimientos para el tratamiento y cuidado del paciente. Coincidiendo con los resultados, su aplicación en áreas como odontología fomenta la capacidad de autoaprendizaje, el análisis crítico y la capacidad adaptativa del profesional (Gerzina et al., 2003). La mejora de la comprensión del problema fomenta el aprendizaje cooperativo. Estudios realizados en matronas destacan la mejora de la dinámica grupal, las habilidades de comunicación, la responsabilidad y el respeto (Rowan et al., 2008). Su aplicación a grupos pequeños en Fisioterapia ha mostrado ventajas frente a métodos tradicionales, pero para que los cambios sean efectivos, el profesor debe aceptar el desafío del cambio curricular (Solomon, 2005). 


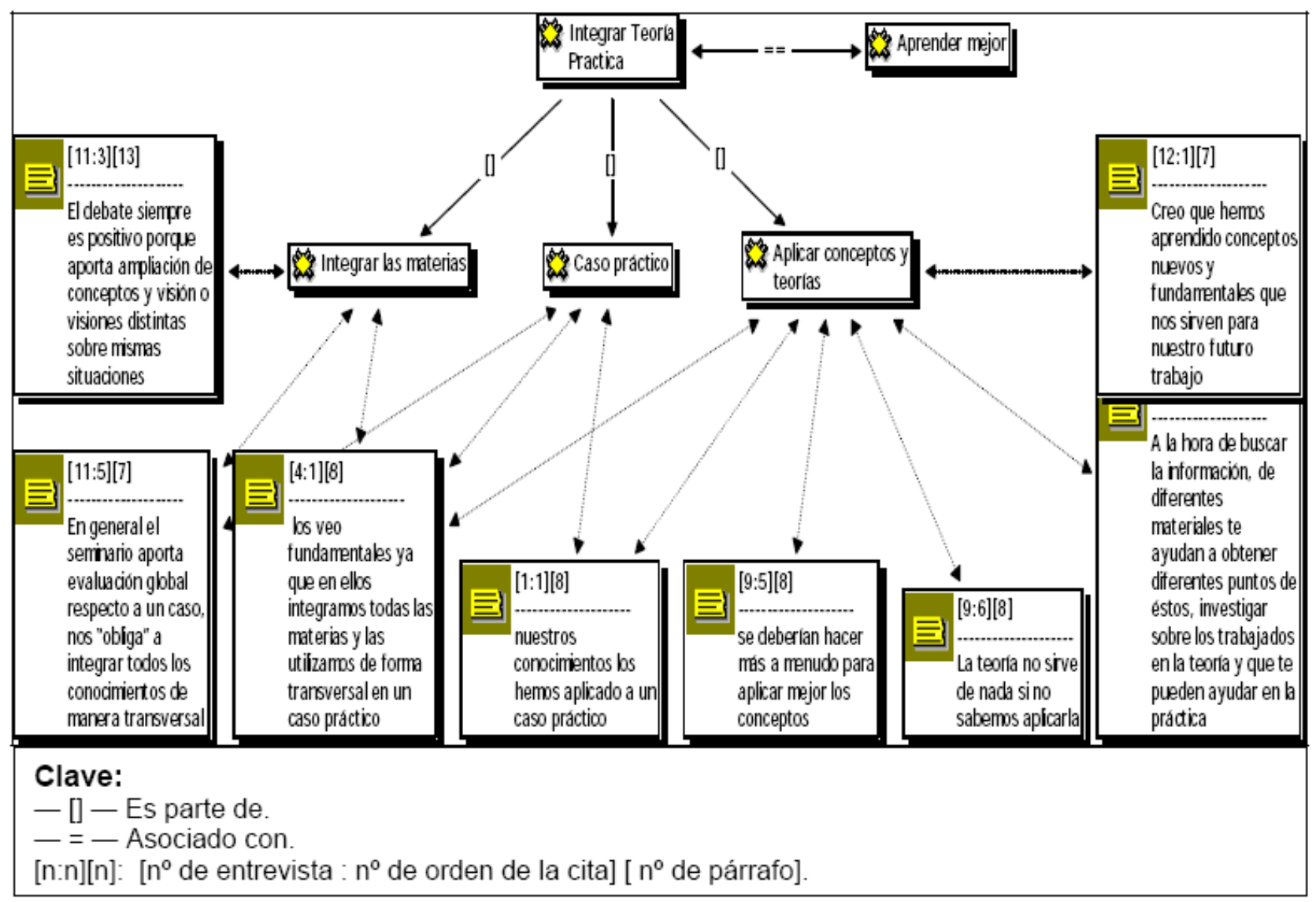

Fig. 1: ABP. Integración teoría-Práctica en CCSS.

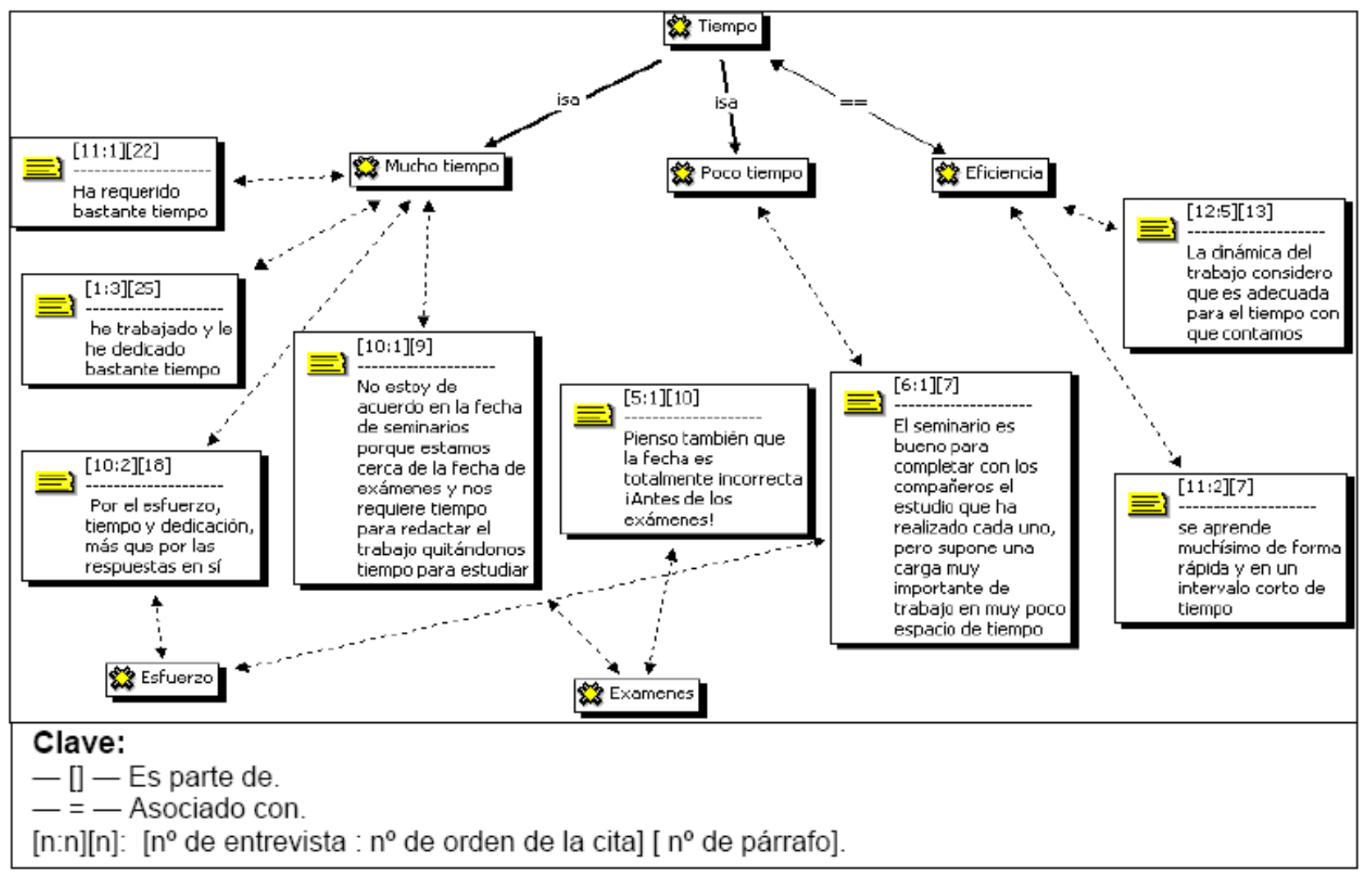

Fig. 2: ABP. Significación para el alumno del tiempo empleado. 
El estudiante lo encuentra más realista, interesante y divertido, implicándose desde el compromiso individual y colectivo. Al igual que en nuestros resultados, la retroalimentación de la información durante el debate (Kang et al., 2009), junto al adecuado tamaño de los grupos (Wells et al., 2009) son esenciales para alcanzar mejor ciertas competencias. En sintonía con otros trabajos, los entrevistados reconocen la importancia del tutor como facilitador del aprendizaje, sobre todo para la detección de lagunas en alumnos principiantes (Mohamad et al., 2010).

La preparación de facilitadores, las guías de tutorización y los talleres, son fundamentales para la consecución de los objetivos (Newton y Wood, 2009). En áreas como la educación del lenguaje, el ABP fomenta la integración teoría-práctica desde un autoaprendizaje permanente, transversal y responsable (Mok et al., 2008); el tutor es clave, pero también sus creencias, valores e interpretación del método ABP (Moore, 2009). En sintonía con otros estudios realizados en medicina (Hueston et al., 2002), también encontramos voces discrepantes con el ABP en cuanto a carencia de evidencias sobre sus ventajas como metodología de enseñanza-aprendizaje en clínica y, si bien parece incrementar la capacidad de toma de decisiones, hay resultados controvertidos en el conjunto de las CCSS (Williams y Beattie, 2008). Y es que la aplicación del $\mathrm{ABP}$ requiere dedicación adicional en cualquier área de conocimiento, no simple aplicación descontextualizada desde el formato tradicional. Su introducción en un plan de estudios implicaría modificar el diseño curricular, el trabajo del alumno y del profesor, los horarios y la evaluación; contribuyendo a eliminar algún malestar del alumnado.

La mejora de resultados no depende de experiencias anteriores (Qin et al., 2010), pero los métodos deben cambiar hacia la autoevaluación, la evaluación formativa y evaluación grupal. Aunque hay evidencias de que la evaluación del ABP es más dificultosa (Smits et al., 2002), ciertos estudios han mostrado la confiabilidad de la evaluación realizada por los propios compañeros (Kamp et al., 2011). Hay que planificar mejor si queremos modificar conocimientos y actitudes, pero sigue habiendo resultados contradictorios en áreas como medicina, que muestran que las diferencias de rendimiento fueron mínimas en el periodo de residencia entre ABP y método tradicional (Distlehorst et al., 2009). Otros estudios realizados apuntan más a incrementos significativos en el bienestar del estudiante de enfermería (Jones y Johnston, 2006), o de la satisfacción, en el de medicina (Smits et al., 2002), que a mejoras en el rendimiento o logro de competencias en la práctica (Applin et al., 2011), no encontrando en el ABP una mejor estrategia educativa. Pero a pesar de ello, esta metodología, desarrollada a través del seminario integrado (Ver Fig. 3), implica para el alumno una serie de cambios de profunda significación en referencia a su proceso de enseñanza-aprendizaje.

\section{CONCLUSIONES}

De lo expuesto y discutido en este trabajo y de los resultados, se puede extraer las siguientes conclusiones principales:

1. La metodología ABP en CCSS, a través del seminario integrado, es valorada positivamente por la mayoría de los alumnos encuestados, significando para ellos una oportunidad de aprendizaje aplicable a la práctica.

2. La responsabilidad en el autoaprendizaje, junto al rol facilitador de los tutores, son entendidas por los alumnos como los elementos claves para la adquisición de competencias a través del ABP.

3. También surgen voces discrepantes que encuentran en el ABP serias limitaciones derivadas, esencialmente, de la descoordinación, la sobrecarga de trabajo y el solapamiento con metodologías tradicionales.

4. Nuestra experiencia evidencia la necesidad de una planificación más profunda, previa a la puesta en práctica de nuevas metodologías de enseñanza y evaluación como el $A B P$, una transición sostenida desde los métodos tradicionales que conduzca más a su complemento que a su sustitución. 


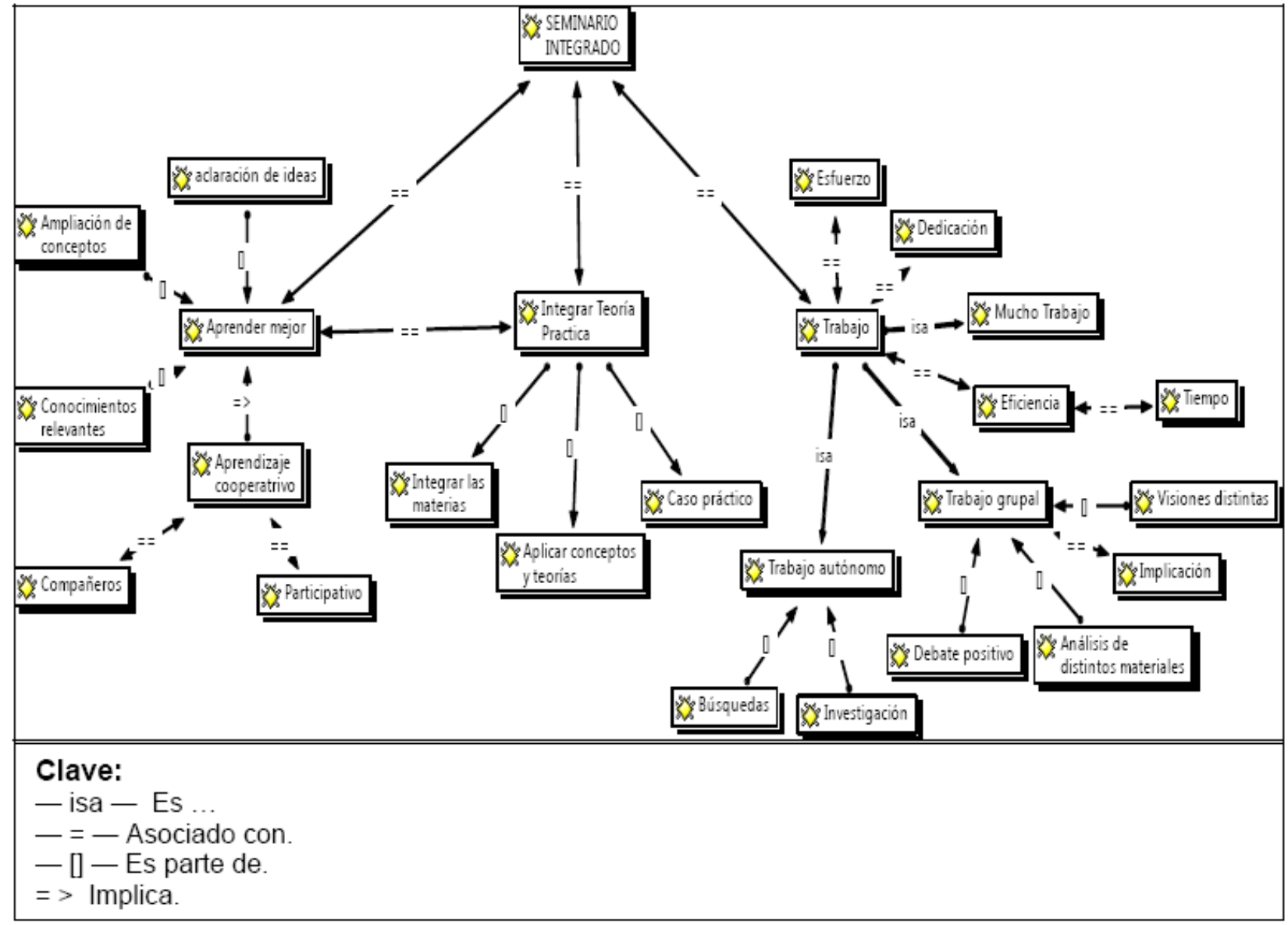

Fig. 3: Seminario integrado. Implicaciones en CCSS.

\section{REFERENCIAS}

Applin, H., Willians, B., Day, R. y K. Buro, A comparison of competencies between problem-based learning and non-problem-based graduate nurses. Nurse Education Today: 31(2), 129-134 (2011).

Barrows, H.S., Taxonomy of problem-based learning methods. Medical education: 20(6), 481-486 (1986).

Burbank, P.M. y D.C. Martins, Symbolic interactionism and critical perspective: divergent or synergistic? Nursing Philosophy: 11(1), 25-41 (2010).

Cancela, A., Sánchez, A., Gandón, R. y M.J. Rey, La gestión de la calidad ante la actual dimensión universitaria en España. Form. Univ.: 3(2), 29-36 (2010).

Charmaz, K., Constructing Grounded Theory. A practical guide through qualitative analysis. Sage, London, (2006).

Chunta, K.S. y E.D. Katrancha, Using problem-based learning in staff development: strategies for teaching registered nurses and new graduates nurses. J Contin Educ Nurs: 41(12), 557-564 (2010).

Distlehorst, L.H., Dawson, B.K. y D.L. Klamen, Supervisor and self-ratings of graduates from a medical school with a problem-based learning and standard curriculum track. Teaching and Learning in Medicine: An International Journal: 21(4), 291-298 (2009).

Fernández, C. y J. Granero, Planificación y guía docente en el marco de la convergencia europea de educación superior. Investigación y Educación en Enfermería: 26(2, sup.), 128-135 (2008).

Flick, U., An introduction to qualitative research. 57 Fourth edition. SAGE, London, (2009).

Gerzina, T.M., Worthington, R., Byrne, S. y C. McMahon, Student use and perceptions of different learning aids in a Problem-Based Learning (PBL) dentistry course. Journal of Dental Education: 67 (6), 641-53 (2003). 
Giuliano, K., Expanding the use of empiricism in nursing: can we bridge the gap between knowledge and clinical practice? Nursing Philosophy: 4(1), 44-52 (2003).

Glaser, G.G. y A.L. Strauss, The Discovery of Grounded Theory: Strategies for Qualitative Research. Aldine, Chicago, (1967).

Hodges, H.F., Preparing new nurses wiht complexity science and problem-based learning. Journal of Nursing Education: 50(1), 7-13 (2011).

Hueston, W.J., Mallin, R. y D. Kern, To what degree do problem-based learning issues change with clinical experience? Teaching and Learning in Medicine: 14(4), 218-222 (2002).

Jonas, H., Técnica, medicina y ética, 22-24. Paidós, Barcelona, (1997).

Jones, M.C. y D.W. Johnston, Is the introduction of a student-centred, problem-based curriculum associated with improvements in student nurse well-being and performance? An observational study of effect. International Journal of Nursing Studies: 43(8), 941-952 (2006).

Kamp, R., Dolmans, D., Van Berkel, H. y H. Schmidt, Can students adequately evaluate the activities of their peers in PBL? Medical Teacher: 33(2), 145-150 (2011).

Kang, W., Jordan E. y M. Porath, Problem-Oriented Approaches in the context of health care education: perspectives and lessons. The Interdisciplinary Journal of Problem-based Learning: 3(2), 43-62 (2009).

Kennedy, T. y L. Lingard, Making sense of grounded theory in medical education. Medical Education: 40(2), 101-108 (2006).

Mohamad, N., Chen, R., Salam, A., Siraj, H.H. y S.Das, Developing skills in problem based learning facilitation: an insight. Int Med J: 17(2), 103-6 (2010).

Mok, C.K., Whitehill T.L. y B.J. Dod, Problem-based learning, critical thinking and concept mapping in speech-language pathology education: a review. International Journal of Speech-Language Pathology: 10(6), 438-48 (2008).

Moore, J., An exploration of lecturer as facilitator within the context of problem-based learning. Nurse Education Today: 29(2), 150-156 (2009).

Morales, P. y V. Landa, Aprendizaje Basado en Problemas. Problem-Based Learning, Theoria: 13 (3), 145-157 (2004).

Newton, Ch. y V. Wood, Reflections on facilitating an interprofessional problem-based learning module. Journal of Interprofessional Care: 23(6), 672-75 (2009).

Qin X.J., Kong, J., Lu, L., Lu, Z.F., y X.K. Wang, Application of problem-based learning in a large class in stomatology course. J Oral Maxillofac Surg: 68(4), 739-43 (2010).

Rowan C.J., McCourth, Ch. y S. Beake, Problem based learning in midwifery- The students' perspective. Nurse Education Today: 28(1), 93-99 (2008).

Schön, D., El profesional reflexivo. Paidós, Barcelona, (1998).

Smits, P., Verbeeck, J. y C. Buisonjé, Problem based learning in continuing medical education: a review of controlled evaluation studies. British Medical Journal: 324 (19 January), 153-156 (2002).

Solomon, P., Problem-based learning: a review of current issues relevant to physiotherapy education. Physiotherapy Theory and Practice: 21(7), 37-49 (2005).

Spencer, J., Learning and teaching in the clinical environment. Brittish Medical Journal: 326 (15 March), 591-594 (2003).

Strauss, A.L. y J. Corbin, Basics of Qualitative Research, 65. Sage, London, (1990).

Wells, H.S., Warelow, P.J. y K.L. Jackson, Problem-Based Learning (PBL): A conundrun. Contemporary Nurse: 33(2), 191-201 (2009).

Williams, S.M. y, H.J. Beattie, Problem based learning in the clinical setting-A systematic review. Nurse Education Today: 28(2), 146-154 (2008).

Wood, F.J., Problem based learning. British Medical Journal: 366(7651), 971 (2008).

Zuberi, R.W., Problem-based learning: where are we now? Medical Teacher: 33(3), 123 (2011). 